\title{
AVALIAÇÃO DAS CONCENTRAÇÕES DE METAIS TRAÇO NOS SEDIMENTOS DO PARQUE DOS MANGUEZAIS, REGIÃO METROPOLITANA DO RECIFE (RMR), PERNAMBUCO, BRASIL.
}

\author{
H. K. P. SILVA ${ }^{1,2}$ \\ S. J. MACEDO ${ }^{2}$ \\ F. M. M. BRAYNER ${ }^{3}$
}

\begin{abstract}
This work quantified trace metals concentrations in sediments samples from an urban area (Mangrove Park), using emission spectroscopy. Average values, for chromium, were 83 $\mathrm{mg} \cdot \mathrm{kg}^{-1}$, in the rainy season, and $82 \mathrm{mg}^{\mathrm{kg}}{ }^{-1}$ in the dry season. For manganese the corresponding average values were $154 \mathrm{mg} . \mathrm{kg}^{-1}$ in and $195 \mathrm{mg} \cdot \mathrm{kg}^{-1}$, and for zinc $255 \mathrm{mg} . \mathrm{kg}^{-}$ ${ }^{1}$ and $224 \mathrm{mg} \cdot \mathrm{kg}^{-1}$. The concentrations for the three metals are above those we measured for background. We conclude that there is contamination by these metals in the study area.
\end{abstract}

Keywords: Trace metals. Estuaries. Sediments. Urban estuary.

\section{INTRODUÇÃO}

Os despejos de resíduos industriais e urbanos lançados nos ecossistemas aquáticos, associados aos processos de urbanização desordenada provocam modificações ambientais como resultado da pressão antrópica nas regiões costeiras. Nestas áreas destacam-se os estuários que vêm sendo objeto de elevado interesse científico, tendo em vista sua importância para um grande número de espécies vivem que neste ecossistema e seu papel como regulador das enchentes.

Os estuários representam ambientes de temperatura e salinidade variáveis, solos lamosos, alta turbidez e topografia irregular. A fauna e a flora têm um alto nível de adaptação evolutiva a condições de estresse, sendo originárias dos ambientes marinhos, fluvial e terrestre (DAY et al., 1989; PRITCHARD, 1967).

Na costa brasileira, nos estuários e deltas dos rios, existe uma estreita faixa de florestas, o manguezal, que é um ecossistema costeiro, característico de regiões tropicais e subtropicais e desempenham um importante papel na distribuição de nutrientes. Além disso, podem atuar como barreiras biogeoquímicas no transporte de metais potencialmente tóxicos, acumulando-os e imobilizando-os no sedimento como por exemplo, $\mathrm{Fe}, \mathrm{Zn}$ e $\mathrm{Pb}$ no ambiente estuarino (DEFEW et al., 2005; SALOMONS et al., 1998).

O lançamento de resíduos contendo ao nível de traços principalmente zinco, cromo cádmio, chumbo têm aumentado sua quantidade nos meios aquático, terrestre e atmosférico, gerando concentrações potencialmente tóxicas, às quais a população pode ser exposta, causando acumulação nos organismos. Esta acumulação pode provocar reações adversas nos organismos como mudanças genéticas, formação de tumores, etc. (AZEVEDO E CHASIN, 2003; PASCALICCHIO, 2002).

Como o sedimento é considerado um reservatório dos contaminantes metálicos lançados no ambiente aquático sua análise passou a ser uma importante ferramenta para indicar o grau de poluição por metais em ecossistemas aquáticos (SILVA, 2001).

Este trabalho avaliou as concentrações dos metais traços cromo, manganês, ferro, cobalto, níquel, cobre, zinco, cádmio e chumbo nos sedimentos do Parque dos Manguezais, região metropolitana da cidade do Recife, determinando suas variações sazonais e o grau

Contatos: ${ }^{1}$ Associação Instituto de Tecnologia de Pernambuco - ITEP/OS; Laboratório de Qualidade de Água - LQA; (Av. Profo Luiz Freire No 700. Cidade Universitária - Recife/PE. CEP. 50740540).

${ }^{2}$ Universidade Federal de Pernambuco - UFPE; Departamento de Oceanografia - UFPE; Programa de Pós-Graduação em Oceanografia; (Av. Arquitetura, S/N. Cidade Universitária - Recife/PE. CEP. 50670-901).

${ }^{3}$ Escola Politécnica de Pernambuco; Programa de Pós-Graduação em Engenharia Civil; (Rua Benfica No 455, Madalena - Recife - PE CEP: 50720-001).

E-mails: helida@itep.br; macedo@ufpe.br; fatimabrayner@upe.poli.br 
do impacto no ecossistema delineando a situação presente. Servirão também como informações de referência para futuros trabalhos de monitoramento na área.

\section{ÁREA DE ESTUDO}

O Parque dos Manguezais, localizado entre as coordenados geográficas de $8^{0} 05^{\prime}$ $05,88^{\prime \prime}$ e $8^{0} 06^{\prime} 40,74^{\prime \prime} \mathrm{S}$, e $34^{\circ} 53^{\prime} 54,66^{\prime \prime}$ e $34^{\circ} 53^{\prime} 58,76^{\prime \prime} \mathrm{W}$, na cidade do Recife Pernambuco, Brasil é um dos últimos resquícios de mangue que ainda restam na cidade que cresceu, desde os seus primórdios, sobre o aterramento destas áreas.

O Parque possui uma área total de 316 hectares, sendo 225,82/ha $(71,26 \%)$ de cobertura vegetal (mangue). É o maior e o mais importante ecossistema aquático do Recife, servindo como controlador do sistema natural de drenagem da cidade caracterizando-se também pela grande quantidade de peixes, crustáceos e moluscos. A área apresenta os manguezais preservados, apesar dos despejos de esgotos e resíduos sólidos em alguns pontos do parque. Muitas ocupações irregulares continuam ocorrendo, e contribuindo para o aumento da poluição e contaminação.

A área possui em seu entorno restaurantes, mini estaleiro, galvânicas, postos de gasolina, gráficas, hospitais e outros estabelecimentos.

Considerando a hidrodinâmica da região entorno do Parque dos Manguezais, Araújo e Pires (1998) realizaram na Bacia do Pina uma simulação exploratória dos efeitos das marés (sizígia e quadratura) na circulação e transporte. Constatou-se que a forma geométrica e a batimetria da referida área têm um efeito significativo, no seu padrão de circulação, e que tais características são úteis na eliminação de poluentes, promovendo um aumento na circulação e no transporte.

No que diz respeito a sua granulometria, os sedimentos do parque apresentaram maior percentual da fração silte/argila (67\%), e variando de areia fina a média (33\%).

\section{MATERIAIS E MÉTODOS}

\section{AMOSTRAGENS}

As amostras superficiais de sedimento foram coletadas nas margens dos rios que cortam o Parque dos Manguezais. As estações de coleta foram distribuídas em 12 pontos ao longo do Parque, em suas margens direita e esquerda (Figura 01), coletadas em uma profundidade até $10 \mathrm{~cm}$, sempre na baixa-mar e posicionadas com o uso de um GPS (Global Position System) modelo Etrex Sumnit Garmin. Durante a realização da coleta foram usadas espátula de aço inox e as amostras foram acondicionadas em sacos plásticos, para posterior tratamento e análise em laboratório. Devido aos parâmetros hidrológicos e com objetivo constatar diferenças sazonais as amostragens foram realizadas durante a estação seca (janeiro e fevereiro de 2007 e 2008) e chuvosa (agosto de 2006 e julho e agosto de 2007).

As amostras de sedimentos usadas como "background" foram coletadas na região interna conhecida como antiga estação de rádio da Marinha do Parque dos Manguezais como descrito acima, no mês de novembro de 2006. 
SILVA; MACEDO; BRAYNER. Avaliação das concentrações de metais traço nos sedimentos do Parque dos Manguezais, Região Metropolitana do Recife (RMR), Pernambuco, Brasil.

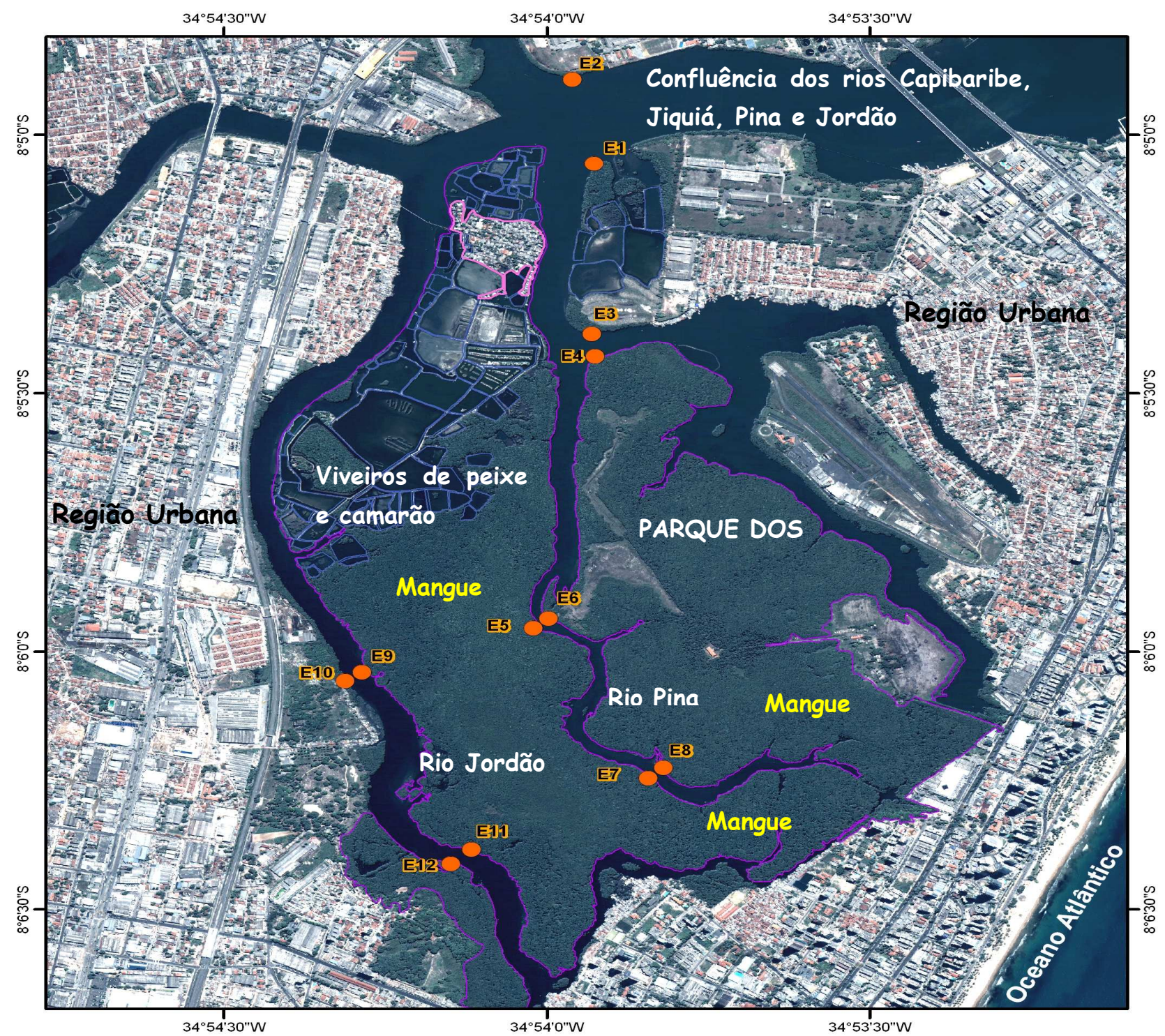

Figura 01. Mapa com a localização das estações de coleta.

FONTE: Imagem cedida pela Unidade de Geoinformação do ITEP/OS, 2009.

\section{PREPARAÇÃO DAS AMOSTRAS PARA ANÁLISE DE METAIS}

A abertura das amostras de sedimento para análise dos metais foi realizada por meio de digestão ácida utilizando um digestor de Microondas DGT 100 Plus da Provecto Analítica e os ácidos nítrico $\left(\mathrm{HNO}_{3}\right)$ p.a., Perclórico $\left(\mathrm{HClO}_{4}\right)$ p.a e Fluorídrico (HF) p.a. de acordo com a metodologia adaptada da Environmental Protection Agency of United States método No 3050B (USEPA, 2001). Com o propósito de garantir a solubilização total das amostras, com a destruição dos compostos silicatados, foi adaptado ao método o uso do HF. Para quantificação das concentrações dos metais foi utilizado um Espectrômetro de Emissão Otica com Plasma Indutivamente Acoplado (ICP-OES), da Spectro, modelo Spectroflame.

\section{CONCENTRAÇÕES BASE OU "BACKGROUND"}

Este trabalho usou como "background" amostras coletadas na própria área de estudo, próximos à estação 04 e 05 na parte interna da antiga Estação de Rádio da Marinha entre as coordenadas $08^{0} 05^{\prime} 58,4^{\prime \prime} \mathrm{S}$ e $34^{\circ} 53^{\prime} 35,01^{\prime \prime} \mathrm{W}$ (TABELA 01).

Tabela 01 - Concentrações médias da área usada como "background".

\begin{tabular}{cccc}
\hline ÁREA & Cr ( $\mathbf{m g} \cdot \mathbf{k g}^{-\mathbf{1}}$ ) & Mn (mg. $\mathbf{~ g}^{\mathbf{- 1}}$ ) & Zn ( $\mathbf{m g} \cdot \mathbf{k g}^{\mathbf{- 1}}$ ) \\
\hline Rádio Pina (interna) (2006) & 25,0 & 160,0 & 41,0 \\
\hline
\end{tabular}




\section{RESULTADOS}

\section{CROMO}

As concentrações médias de cromo, no sedimento superficial da área em estudo, oscilaram entre $25 \mathrm{mg} \cdot \mathrm{kg}^{-1} \mathrm{em}$ fevereiro/2007 nas estações 04 e 08, no período seco e 231 $\mathrm{mg} \cdot \mathrm{kg}^{-1}$ na estação 12 no mês de agosto/2007, no período chuvoso, apresentando um valor médio de $82 \pm 21 \mathrm{mg} \cdot \mathrm{kg}^{-1}$ e de $80 \pm 16 \mathrm{mg} \cdot \mathrm{kg}^{-1}$ durante o período chuvoso e seco, respectivamente (FIGURA 02 ).

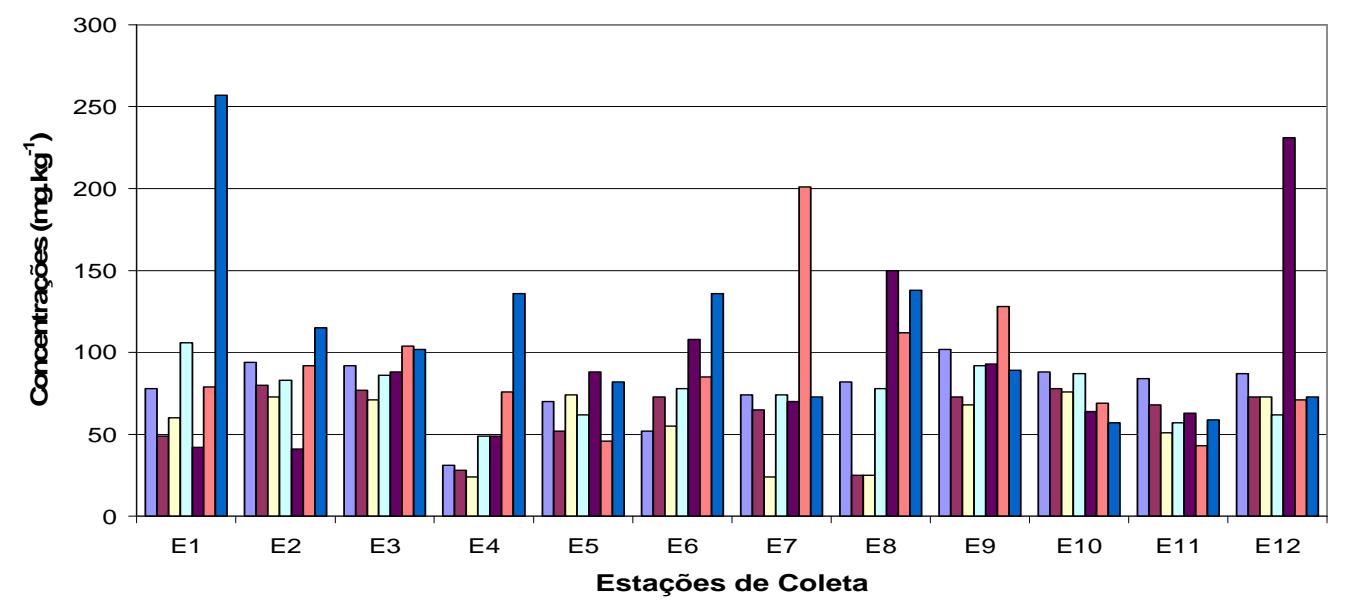

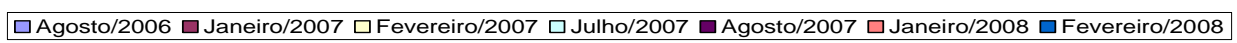

FIGURA 02. Concentrações médias de cromo no sedimento superficial.

\section{MANGANÊS}

As concentrações médias de manganês oscilaram entre $91 \mathrm{mg} \cdot \mathrm{kg}^{-1} \mathrm{em}$ agosto/2007 na estação 06 , no período chuvoso e $338 \mathrm{mg} \cdot \mathrm{kg}^{-1}$ na estação 08 no mês de fevereiro/2007, no período seco, com valor médio de $171 \pm 19 \mathrm{mg} \cdot \mathrm{kg}^{-1}$ no período chuvoso e de $189 \pm 21$ $\mathrm{mg} \cdot \mathrm{kg}^{-1}$ no período seco (FIGURA 03).

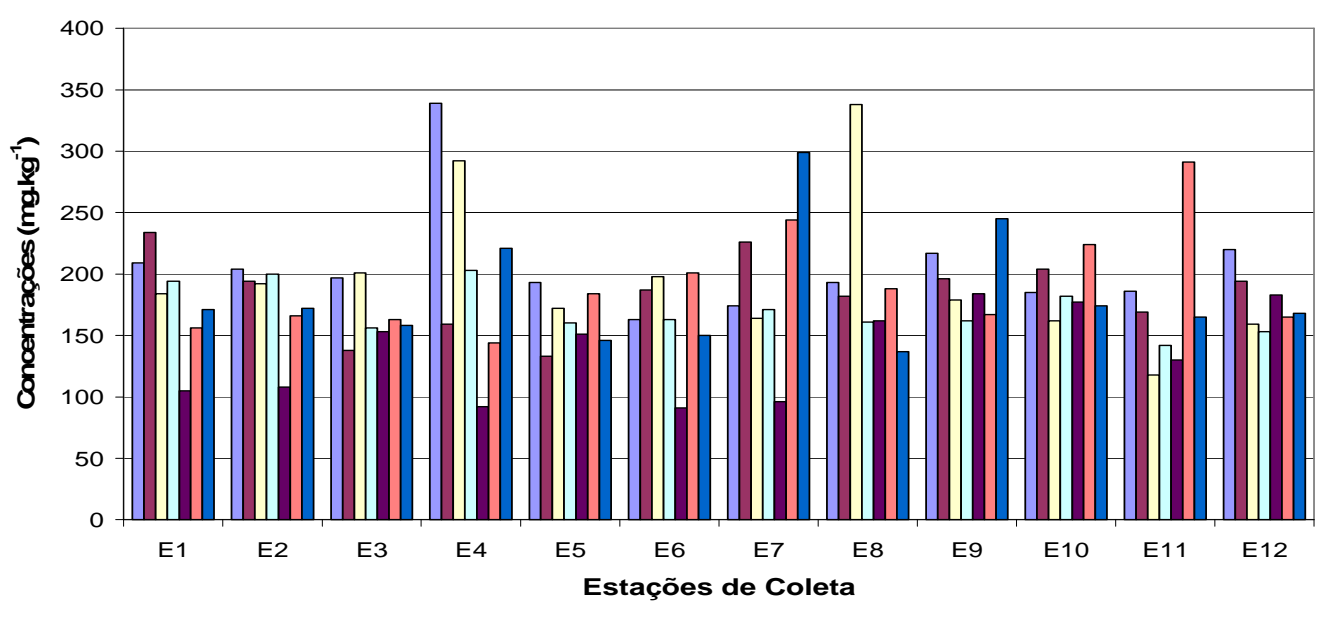

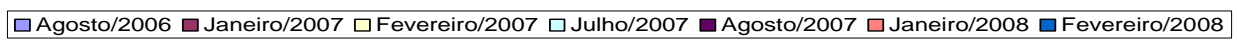

FIGURA 03. Concentrações médias de manganês no sedimento superficial.

\section{ZINCO}

As concentrações médias de zinco oscilaram entre $75 \mathrm{mg} \cdot \mathrm{kg}^{-1}$ em fevereiro/2007 na estação 07, no período seco e $613 \mathrm{mg} \cdot \mathrm{kg}^{-1}$ na estação 05 no mês de agosto/2007, período chuvoso, com valores médios de $285 \pm 109 \mathrm{mg} \cdot \mathrm{kg}^{-1}$ no período chuvoso e de $225 \pm 68$ $\mathrm{mg} \cdot \mathrm{kg}^{-1}$ no período seco (FIGURA 04). 
SILVA; MACEDO; BRAYNER. Avaliação das concentrações de metais traço nos sedimentos do Parque dos Manguezais, Região Metropolitana do Recife (RMR), Pernambuco, Brasil.

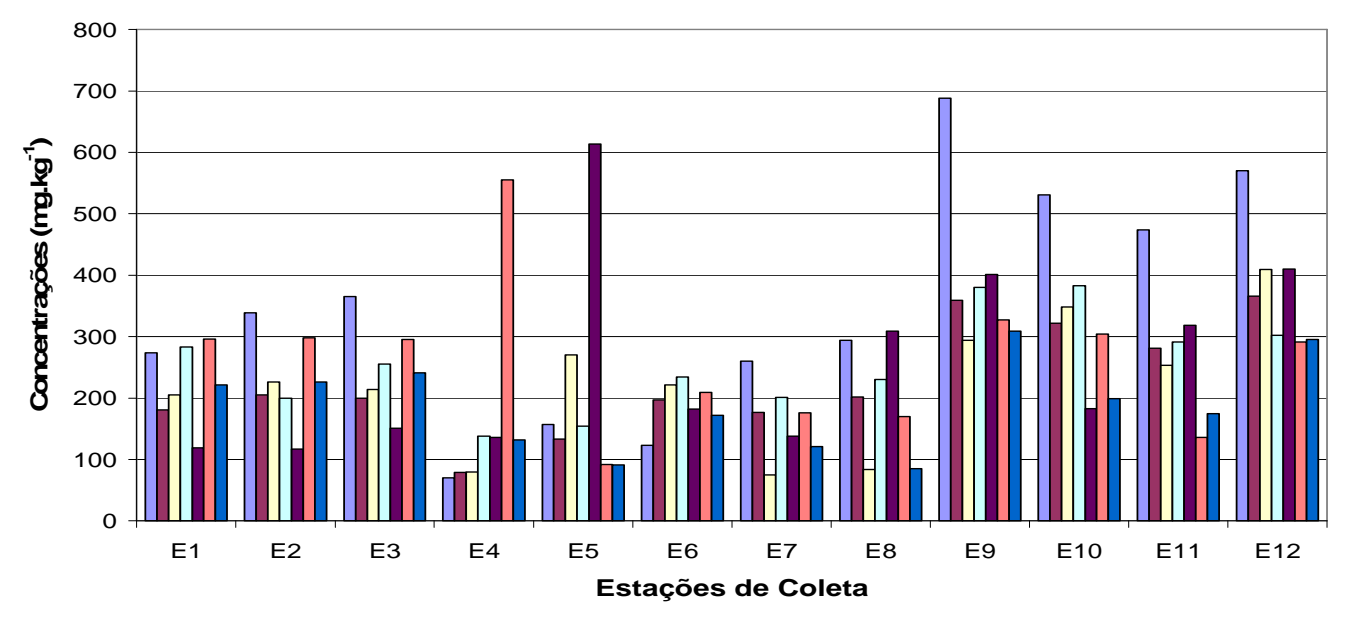

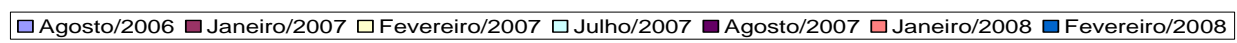

FIGURA 04. Concentrações médias de zinco no sedimento superficial.

\section{FERRO}

As concentrações médias de ferro oscilaram entre $11.500 \mathrm{mg} \cdot \mathrm{kg}^{-1} \mathrm{em}$ janeiro/2007 na estação 04, no período seco e $43.120 \mathrm{mg}^{\mathrm{kg}}{ }^{-1}$ na estação 03 no mês de julho/2007, no período chuvoso, com valores médios de $32.174 \pm 5.345 \mathrm{mg} \cdot \mathrm{kg}^{-1}$ no período chuvoso e de $26.139 \pm 3.291 \mathrm{mg} \mathrm{kg}^{-1}$ no período seco (FIGURA 05).

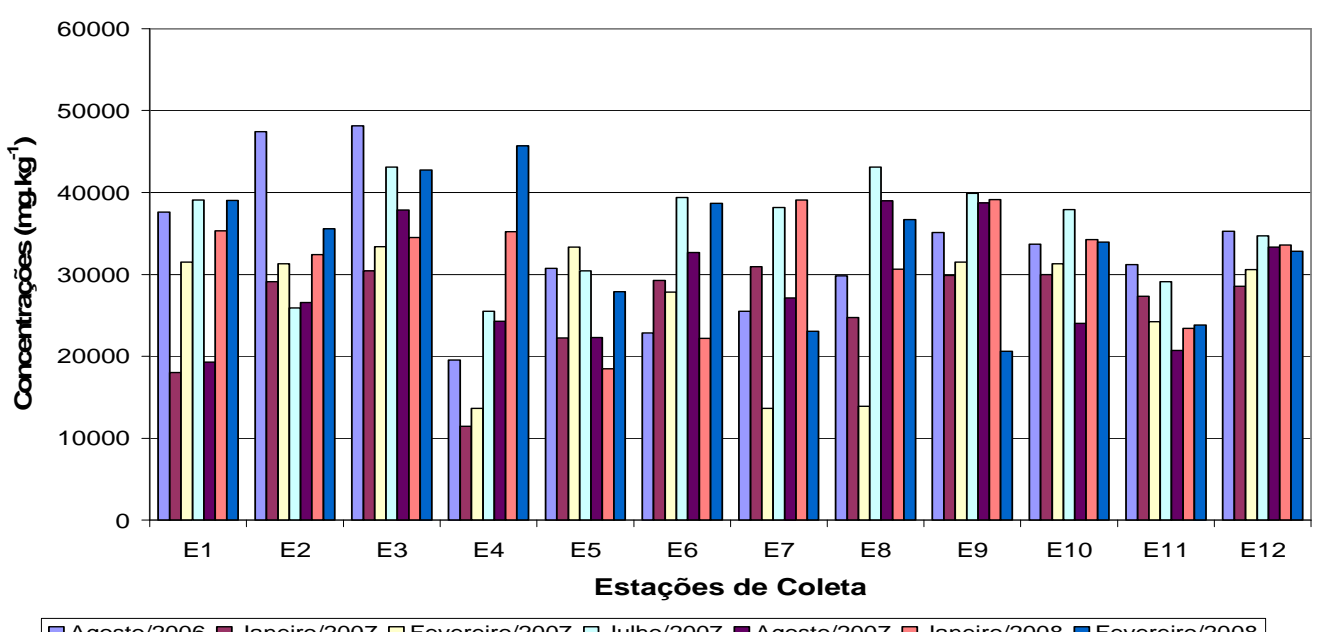

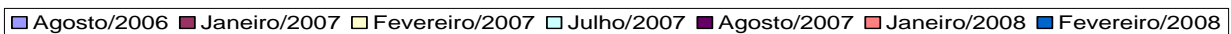

FIGURA 05. Concentrações médias de ferro no sedimento superficial.

\section{DISCUSSÃO}

Analisando e comparando os resultados obtidos de cromo, manganês e zinco com o "background" proposto, foi observado que os valores das concentrações destes metais nos sedimentos obtidos para o Parque dos Manguezais estão acima dos que foram encontrados na área de referência, indicando contaminação em todas as estações, oriunda das diferentes fontes poluidoras, como galvânicas, mini estaleiros, efluentes domésticos e outros.

Segundo Lee (1980) o cromo é largamente empregado em processos de cromação, no curtimento de couros, na anodização do alumínio e como agente oxidante. Os resultados obtidos para cromo nos sedimentos no Parque dos Manguezais indicaram que estes se encontram contaminados e que devido à geomorfologia da região, ações antrópicas há um maior aporte deste metal na estação 08.

O manganês possui propriedades químicas onde pequenas mudanças na variação de $\mathrm{pH}$, potencial redox, variações de oxigênio dissolvido e salinidade, contribuem de forma efetiva no aumento de sua disponibilidade para o ambiente. As variações características dos estuários permitem que este metal ocorra nos seus diferentes números de oxidação, contribuindo para a formação de óxidos (LACERDA et al, 1993; CALMANO et al., 1993; SWADDLE, 1997; BRAYNER e MATVIENKO, 2003). Os manguezais oferecem condições para 
que essas propriedades químicas do manganês ocorram podendo ser observadas nos resultados obtidos onde se tem uma maior concentração deste metal no período seco, indicando a importância do estudo sazonal para este elemento (LEE, 1980).

O zinco possui características específicas de seu grupo, como por exemplo, ligações fracas, que o disponibilizam facilmente para o ambiente (Lee, 1980), fato este comprovado por Brayner et al. (2001) que realizaram a especiação de metais pesados em sedimentos em uma área estuarina do rio Capibaribe e constataram que nas primeiras etapas da especiação o zinco foi encontrado em concentrações bastante próximas às encontradas neste trabalho, que em todas as estações durante o período estudado apresentaram valores superiores ao "background" indicando contaminação por zinco nos sedimentos da área estudada, especialmente nas estações 04, 05, 09, 10 e 12 cuja concentração foi superior a $500 \mathrm{mg} \cdot \mathrm{kg}^{-1}$. Esse aumento se deu devido a remoção dos sedimentos para a construção de viveiros de camarão.

Deve-se ressaltar que os resultados obtidos para o cromo, manganês e zinco nos sedimentos do Parque dos Manguezais ratificaram os trabalhos desenvolvidos, no estado de Pernambuco, em sedimentos de viveiros de cultivo de peixes por Brayner (1998), Brayner et al. (2001); em sedimentos estuarinos no rio Capibaribe, por Silva (2004), Lira (2008) ao estudá-los na Lagoa do Araçá e Noronha (2008) no estuário do rio Timbó.

Os trabalhos desenvolvidos na área estudada, contribuíram de maneira direta, para demonstrar a importância da determinação de metais traço em áreas urbanas, fornecendo um excelente indicador químico para estabelecimento de um sistema de monitoramento adequado a um perfeito estudo sobre a contaminação de regiões estuarinas.

Quanto ao ferro o estado de oxidação $\left(\mathrm{Fe}^{2+}\right.$ e $\mathrm{Fe}^{3+}$ ) e suas propriedades físicas e químicas determinam sua disponibilidade no meio ambiente e biota. As condições anóxicas proporcionam condições para que o ferro sofra mudanças no número de oxidação em seu contato com a coluna d'água, disponibilizando-o ou não para o ecossistema (AZEVEDO E CHASIN, 2003). Tais características podem ser constatadas na região do Parque dos Manguezais e podem ser observadas nos resultados obtidos para concentração de ferro neste estudo.

Desta maneira pode-se afirmar que os sedimentos do estuário em pauta podem ser usados para um monitoramento específico de metais traço na área.

Nota-se uma grande variabilidade nas concentrações encontradas no conjunto das estações de coleta, em torno dos $50 \%$, tanto para o período seco como para o chuvoso. Ela é surpreendente em um sistema como o sedimento que por crescer lentamente deveria ser uniforme sobre esta área de morfologia similar. Uma possível causa da não uniformidade é ação antrópica não uniforme no passado.

\section{CONCLUSÕES}

De acordo com os resultados obtidos, pode-se concluir que:

1. Os sedimentos do Parque dos Manguezais apresentaram concentrações de metais acima dos valores obtidos de "background", para cromo, manganês e zinco, indicando contaminação na área de estudo.

2. Os sedimentos estudados podem ser usados como base para um monitoramento específico de metais traço na área, refletindo de uma maneira geral na qualidade do ecossistema manguezal e que os sedimentos apresentaram-se como importantes compartimentos que armazenam zinco, cromo e manganês.

3. Quanto à variabilidade sazonal do sedimento se conclui que as concentrações encontradas de metais são estatisticamente indistinguíveis nos períodos chuvosos e secos.

\section{AGRADECIMENTOS}

Os autores desejam expressar seus agradecimentos ao Conselho Nacional de Desenvolvimento Científico e Tecnológico (CNPq), pelo auxílio financeiro que possibilitou a realização desta pesquisa. 
SILVA; MACEDO; BRAYNER. Avaliação das concentrações de metais traço nos sedimentos do Parque dos Manguezais, Região Metropolitana do Recife (RMR), Pernambuco, Brasil.

\section{REFERÊNCIAS}

AZEVEDO, F.A.; CHASIN, A. A. M. (2003). Metais: gerenciamento da toxicidade. São Paulo: Editora Atheneu. 554p.

BRAYNER, Fátima Maria Miranda. (1998). Determinação de taxas de retenção de metais-traço por sedimentos orgânicos em um viveiro de piscicultura em área estuarina e urbana. São Carlos: USP/EESC, 1998. Tese de doutorado, Universidade de São Paulo.

BRAYNER, F. M. M.; SILVA, H. K. P.; BARBOSA, A. M. F. (2001). Speciation of heavy metals in estuarine sediments in the Northeast of Brazil. Environ Sci 8 Pollut Res 8 (2001). p. 1-6. August.

BRAYNER, F. M. M.; MATVIENKO, B. (2003). Manganese and iron as oxygen carriers to anoxix estuarine sediment. Journal de Physique IV. France 107.p. 221-225.

DAY, J. W. et al. (1989). Estuarine ecology. New York, USA: Wiley. 558 p.

LEE, John David. (1980). Química inorgânica: um novo texto conciso. 3. ed. São Paulo: Edgard Blucher. 508 p.

LIRA, Josineide Braz de Miranda. (2008). Avaliação preliminar das concentrações de metais pesados em sedimentos da Lagoa do Araçá, Recife - Pernambuco, Brasil. Dissertação de mestrado (ITEP/OS). Recife.

NORONHA, Tibério Jorge Melo de. (2008). Avaliação das concentrações de metais pesados em sedimentos do estuário do rio Timbó, Pernambuco - Brasil. Dissertação de mestrado (ITEP/OS). Recife.

PASCALICCHIO, E. A. (2002) Contaminação por metais pesados. São Paulo: Editora Annablume. 132p.

PRITCHARD, D. W. (1967) What is an estuary, physical viewpoint. In: G. H. Lauf (editor): Estuaries. American Association for the Advancement of Science, Washington D.C., publ. no. 83, 1989.

SALOMONS,W.; FÖRSTNER, V. (1984). Metals in hydrocycle. Berlin: Spring Verlag. 349 p.

SALOMONS, W. (1998). Behavior and impact assessment of heavy metals in estuarine and coastal zones. In: SEELIGER, U.; LACERDA, L. D. ; PATCHINEELAN, S. R. metals in Coastal Environment of Latin America. Berlin, Sringer-Verlag.

SILVA, C. A. R. (1998). Distribuição e ciclagem interna de metais pesados em um ecossistema de manguezal combinado com Rhizophora mangle, Baía de

Sepetiba, Rio de Janeiro. Niterói: UFF, 1998. Dissertação de mestrado, Universidade Federal Fluminense.

SILVA, Hélida Karla Philippini da Silva. (2001). Especiação de Metais: Uma ferramenta para educação ambiental. Recife: UFRPE, 2001. Monografia de graduação, Universidade Federal Rural de Pernambuco.

SILVA, Hélida Karla Philippini da Silva. (2004). Concentrações dos metais pesados nos sedimentos do estuário do rio Capibaribe, Região Metropolitana do Recife (RMR) Pernambuco, Brasil. Recife: UFPE, 2004. Dissertação de mestrado, Universidade Federal de Pernambuco.

SILVA, Hélida Karla Philippini da Silva. (2010). Avaliação das Concentrações de Metais Traço e Suas Interações nos Sedimentos e Biota do Parque dos Manguezais, Região Metropolitana do Recife (RMR) - Pernambuco, Brasil. Recife: UFPE, 2010. Tese de Doutorado, Universidade Federal de Pernambuco.

USEPA. (2001). Methods for collection, storage and manipulation of sediment for chemical and toxicological analyses: technical manual. EPA 823-B-01-002. v. 1. Method 3050B. Envirinmental Protection Agency, Office of Water, Washington, DC. 